\title{
Kernos
}

Revue internationale et pluridisciplinaire de religion grecque antique

$29 \mid 2016$

Varia

\section{Les Rédacteurs grecs d'enquêtes sur le passé héroïque : ni mythographes, ni mythographie}

\section{Claude Calame}

\section{(2) OpenEdition \\ Journals}

Édition électronique

URL : http://journals.openedition.org/kernos/2413

DOI : 10.4000/kernos.2413

ISSN : 2034-7871

Éditeur

Centre international d'étude de la religion grecque antique

Édition imprimée

Date de publication : 1 octobre 2016

Pagination : 403-414

ISSN : 0776-3824

Référence électronique

Claude Calame, «Les Rédacteurs grecs d'enquêtes sur le passé héroïque : ni mythographes, ni

mythographie », Kernos [En ligne], 29 | 2016, mis en ligne le 01 octobre 2016, consulté le 18 novembre 2020. URL : http://journals.openedition.org/kernos/2413; DOI : https://doi.org/10.4000/kernos.2413

Ce document a été généré automatiquement le 18 novembre 2020.

Kernos 


\title{
Les Rédacteurs grecs d'enquêtes sur le passé héroïque : ni mythographes, ni mythographie
}

\author{
Claude Calame
}

\section{RÉFÉRENCE}

Robert L. FOWLER, Early Greek Mythography. Volume 1: Text and Introduction, Oxford, OUP, 2001. $18 \times 25 \mathrm{~cm}, 508$ p. ISBN : 978-0-19-814740-4 ; Volume 2: Commentary, Oxford, OUP, 2013. $18 \times 25 \mathrm{~cm}, \mathrm{xxi}+825$ p. ISBN : 978-0-19-814741-1.

1 La mythographie grecque constitue-t-elle un genre discursif? C'est la question à laquelle se trouve d'emblée confronté l'éditeur des deux volumes de Early Greek Mythography, édition commentée des fragments des «mythographes » grecs. De la définition donnée autant à la pratique de la mythographie qu'au statut de mythographe dépendent les critères qui permettent de délimiter, sous l'étiquette de "mythography», le corpus des fragments méritant édition et commentaire. Ces critères sont à la fois de l'ordre du " genre » (discursif) et d'ordre chronologique. Or le problème de définition et de délimitation du corpus est double dans la mesure où ni muthográphos ni muthographía ne sont des termes attestés pour la période recouverte par l'ensemble proposé (sous la qualification de " early ») : de la fin du $\mathrm{VI}^{\mathrm{e}} \mathrm{s}$. au début du IV ${ }^{e}$ s. av. n.è. (R.F., p. XXXIII) ${ }^{1}$.

\section{« Mythographes » : une désignation tardive}

2 Muthográphos apparaît dans notre tradition, à l'évidence très lacunaire, par exemple chez Polybe (IV, 40, 1-2), au II ${ }^{\mathrm{e}}$ siècle avant l'ère chrétienne. Les mythographes y sont associés aux poètes ; leurs explications quant au courant qui s'écoule du Pont-Euxin et de son comblement par des alluvions ne sauraient résister à « l'observation selon la 
nature " (he katà phúsin theoría). Se réclamant d'Héraclite (fr. 22 A 23 Diels-Kranz) et s'écartant de ses propres prédécesseurs (dénommés " rédacteurs " : suggrapheîs), Polybe lui-même gagnera la confiance de ses auditeurs (sic!) par l'exactitude de son enquête (historía), dans le moment présent. Polybe (III, 91, 7) offre un emploi analogue de muthográphos, également dans un contexte géographique, à propos des Champs Phlégréens! Avant le $\mathrm{II}^{\mathrm{e}}$ siècle la seule attestation du mot se trouve au terme de l'une des Histoires incroyables recueillies par un Palaiphatos à la tradition biographique multiple. Du récit de la résurrection de Glaucos après sa mort supposée dans un pithos rempli de miel (26), Palaiphatos nous dit qu'il est à l'origine du mûthos façonné par les mythographes; en raison de l'impossibilité pour un homme de revivre, il en donne une version rationalisante. Le lógos serait-il opposé au mûthos qui apparaîtrait comme le propre des mythographes? À vrai dire, il est loin d'être certain que le texte du double récit sur Glaucos tel qu'il nous est parvenu appartienne au recueil d'origine, que l'on date en général de la seconde moitié $d u \mathrm{IV}^{\mathrm{e}}$ siècle $^{2}$. Par ailleurs, dans le prélude du recueil, Palaiphatos dit tirer les récits (tà legómena) qu'il rationalise de poètes et de logographes qu'il désigne aussi comme suggrapheîs; de même dénomme-t-il par deux fois suggráphein sa pratique narrative de réécriture, qui est le résultat d'une enquête (historésas).

3 De manière générique, au pluriel, le terme est assez souvent employé un siècle après Polybe par Diodore de Sicile. L'historien attribue à certains parmi les « mythographes », par exemple, une version particulière de l'épisode de la descente de Thésée et de Pirithoos dans l'Hadès (IV, 63, 4) ou encore l'hypothèse que la Sicile était à l'origine une presqu'île (hoi palaioì muthográphoi; IV, 85, 3). Quant à l'histoire des héros qui ont été immortalisés tels Héraclès, Dionysos ou Aristée, elle fait l'objet de récits transmis aussi bien par des historiens que par des mythographes (lógoi parà toîs historikoîs te kaì muthográphois): parmi les historiens, Évhémère de Messène; au nombre des «mythologues» (le terme est sans doute emprunté à Platon; cf. République, 392 d), Homère, Hésiode et Orphée, c'est-à-dire des poètes qui ont façonné au sujet des dieux des récits (mûthoi) pleins de miraculeux. Les mythographes sont aussi associés aux poètes (anciens) par Denys d'Halicarnasse dans les Antiquités Romaines (I, 13, 2) à propos de l'origine du peuple indigène des Oinôtroi qu'on va retrouver en évoquant la figure d'Antiochos de Syracuse.

4 Mais, en progressant dans le temps, il faut naturellement mentionner le fameux prélude qui ouvre la Vie de Thésée de Plutarque (1,1-5). Appartenant au domaine des temps plus anciens que ceux qui peuvent faire l'objet d'une enquête sur les actes et d'un discours vraisemblable (eikóti lógoi), la biographie du grand héros athénien sera épurée de son muthôdes par le travail même de l'enquête (historía, à trois reprises). Quant au merveilleux de ce temps des héros, Plutarque non seulement associe à son tour les mythographes aux poètes; mais surtout il compare les " histoires tragiques " (tragiká) dont ils traitent, sans inspirer la moindre confiance, aux régions inhabitées et sauvages auxquelles sont confrontés les enquêteurs (historikoí) géographes quand ils parviennent aux confins de la terre habitée.

5 Ainsi donc, les « rédacteurs de mythes ", dès le $\mathrm{II}^{\mathrm{e}}$ siècle avant l'ère chrétienne, sont des rédacteurs de récits relatifs à un passé ancien ou à des régions éloignées. 


\section{La tardive « mythographie »}

6 De muthographía, on ne trouve pas d'emploi avant Strabon, au $\mathrm{I}^{\mathrm{er}}$ siècle de l'ère chrétienne ${ }^{3}$. En particulier à propos d'êtres fabuleux tels les Macrocéphales, les Hémicynes ou les Pygmées, le géographe relève une confusion fréquente entre ce qui relève du muthikón et ce qui est de l'ordre de l'historikón (I, 2, 35). Aux poètes tels Homère, Hésiode ou Alcman (le premier mutheúon, le dernier historôn!) sont comparés ceux qui rédigent en prose (hoi pezêi suggráphontes) sous la forme de l'enquête (en historías skhémati) ; par la manière prosaïque, les seconds effaceraient les effets d'une rédaction sous la forme du mythe (muthographía) qui, de ce fait, est attachée implicitement et paradoxalement à la poésie. Et si les prosateurs tissent des fables relatives à des domaines obscurs et inconnus, ce n'est pas par ignorance de ce qui est, mais c'est pour séduire leur public par un merveilleux qu'ils ont façonné. C'est ce que font Hérodote, Ctésias, Hellanicos (FGrHist 4 T 19 = test. 19 Fowler) et les rédacteurs d' Indica. Théopompe lui aussi raconte des «mythes" dans ses «histoires", mais il le reconnaît et l'affirme explicitement (cf. FGrHist 115 F 381). Par ailleurs, au sujet des Épéens, habitants de l'Élide dans le Catalogue des vaisseaux de l'Iliade (II, 615-619), plusieurs versions circulent - nous dit Strabon (VIII, 3, 9) - quant à leurs rapports avec les Éléens. Selon Hécatée ( FGrHist 1 F 121 = fr. 25 Fowler) par exemple, ils auraient aidé Héraclès à se rendre maître d'Augias et de l'Élide. Mais le géographe ajoute que ces anciens rédacteurs (arkhaîoi suggrapheîs) racontent (légousin) beaucoup de choses qui ne correspondant pas à ce qui est; leur désaccord s'expliquerait par une habitude du mensonge qui est congénitale aux « mythographies».

7 Quant à muthográphein, l'un des rares usages du verbe est localisable également chez Strabon (III, 4, 4). Le verbe y désigne la narration que fait Homère (en diction poétique) des voyages d'Ulysse. Si le poète a situé la majorité des stations du héros au-delà des Colonnes d'Hercule, c'est que les données de l'enquête (tà historoúmena) sont proches d'une fabrication (plásma) qui, de ce fait, n'est pas dépourvue de créance ! Voilà donc la poésie épique jugée à l'aune des critères de l'enquête géographico-historique.

\section{Anciens enquêteurs et pratiques de l'écriture}

Assurément, R.F. procède à une analyse lexicale et sémantique (I, p. XXVIII-XXXII) autour du concept de "mythography ", mais elle est à fois incomplète et fautive du point de vue de la méthode linguistique. Ignorée, la distinction opératoire entre signifiant et signifié, et par conséquent entre champ lexical (termes morphologiquement associés à une racine) et champ sémantique (termes dont le sens est proche en dépit de morphologies totalement différentes) est pourtant ici essentielle.

Du point de vue du champ lexical, la fonction des auteurs individuels inclus dans le corpus défini par R.F. n'est pas désignée par le terme muthográphos. Pour ne prendre que cet exemple, Hécatée, dans la notice que lui consacre la Souda (s.v. Hekataîos, e 360 Adler), est présenté comme historiográphos. On se rappelle par ailleurs qu'Hérodote (II, $143,1 ; \mathrm{V}, 36,2 ; \mathrm{V}, 125,1)$ fait régulièrement de son collègue un simple logopoiós alors qu'il est offert comme le modèle du historikós par Élien (parallèlement à Pythagore comme sophós, Olympos comme mousikós et Homère comme poète ; VH, 13, 20). Mais le même Élien $(\mathrm{VH}, 9,23)$ qualifie Hécatée de logopoiós dans un passage où il compte l'historiographe parmi les poètes et ceux qui composent des arkhaîoi mûthoi. Enfin 
Strabon, dans le passage déjà cité à propos de la muthographía (VIII, 3, 9), compte Hécatée parmi les arkhaîoi suggrapheîs. On se souvient que, sous le calame de Polybe (IV, $40,1)$, c'est aussi le terme de suggrapheîs qui englobe au pluriel les «mythographes » prédécesseurs de l'historien lui-même sur la question des raisons à donner au courant s'échappant du Pont Euxin. C'est donc là la dénomination la plus courante de rédacteurs qui n'ont de «mythographe » que le nom moderne. Dans le petit traité qu'il consacre à Thucydide $(5,1-2)$, Denys d'Halicarnasse désigne comme arkhaîoi suggrapheîs non seulement Hécatée, mais aussi Eugéon de Samos, Charon de Lampsaque, Eudémos de Paros ou Acousilaos d'Argos. Ces « anciens rédacteurs ", qui ont précédé Thucydide, sont dits avoir rédigé des enquêtes sur les Grecs et sur les barbares, par peuples et par cités, en se fondant sur des traditions orales et sur des documents archivés dans des temples ou des lieux profanes; sans doute ces enquêtes contiennent-elles des mûthoi, mais ce sont des récits auxquels on accorde foi depuis longtemps. Ils appartiennent donc à une tradition acceptée.

De ces désignations d'Hécatée compris dans sa «fonction-auteur ", la liste complète est d'ailleurs dressée par R.F. lui-même (I, p. 110), mais sans qu'il en tire la conclusion qui s'impose : au sens grec du terme, Hécatée n'est pas un mythographe! Ajoutons que, du côté latin, un Hellanicos de Lesbos, pratiquement contemporain d'Hérodote et de Thucydide, est dénommé historiae scriptor précisément en association avec ces deux historiographes (Aulu-Gelle, XV, 23) alors que la Chronique d'Eusèbe (Ol., 70, 1, p. 107 c Helm) en fait un historiografus voisin du filosofus Démocrite et du physicus Anaxagore. Du côté grec, Hellanicos est considéré comme historikós (en particulier par la notice de la Souda, s.v. Hellánikos, e 739 Adler) ou comme (arkhaîos) suggrapheús, par exemple par Strabon (XIII, 2, 4) qui le compte également au nombre des palaioi suggrapheîs (en XI, 6, 2). Denys d'Halicarnasse (Thuc., 5, 2) qui le place parmi les arkhaîoi suggrapheîs déjà mentionnés, dans un série qui, proche de Thucydide, comprend encore Damastès de Sigée, Xénomédès de Céos et Xanthos de Lydie. La liste (presque) exhaustive des désignations indigènes d'Hellanicos de Lesbos est également donnée par R.F. (I, p. 147). On consultera sur ces dénominations indigènes le très utile index des Verba potiora ex auctoribus (I, p. 456-458, avec les renvois de rigueur aux textes enregistrés comme testimonia). Pas de trace de muthográphos!

Du point de vue des pratiques d'écriture, rappelons d'abord que, dans le prélude de son enquête qui assume la forme d'une sphragís, le même Hécatée de Milet désigne sa parole d'autorité par le verbe mutheîsthai. À la troisième personne, comme il se doit dans la procédure de la "signature » (qu'elle soit poétique ou prosaïque), cette forme verbale est accompagnée d'un geste de deixis verbale exprimé par l'adverbe démonstratif hôde. Ainsi désignée, la rédaction écrite de l'enquête - on s'en souvient- est formulée comme un acte non pas de parole, mais d'écriture, également de manière déictique : táde grápho, «j'écris ceci ». Donné comme acte de vérité, cet acte scripturaire est posé en face des autres récits des Grecs qui sont désignés quant à eux par le terme lógoi ; ce sont des discours qui lui semblent risibles ${ }^{4}$. D'Antiochos de Syracuse, nous avons aussi une "signature » dans laquelle le geste de deixis verbale désignant le discours qui va suivre est subordonné au terme plus technique de suggráphein : táde sunégrapse (FGrHist 555 F 2 = fr. 2 Fowler); parmi les anciens récits (arkhaîoi lógoi), l'historiographe déclare ne reconfigurer par écrit que les plus dignes de foi et les plus assurés. Denys d'Halicarnasse, par deux fois (Ant. Rom. I, 12, 3 et 73, 3), fait d'Antiochos un suggrapheús ; de même en va-t-il de Diodore de Sicile (XII, 71, 2). Ce terme n'est autre que celui par lequel Thucydide inaugure son récit : dans sa propre signature initiale, il désigne ainsi 
sa pratique de composition écrite (sunégrapse) quant au déroulement de la "guerre que se déclarèrent les uns aux autres les Péloponnésiens et les Athéniens ».

\section{La mythographie comme genre discursif ?}

Mais qu'en est-il de la mythographie comme éventuel genre (discursif)? On le sait, l'ouvrage d'Hécatée (en quatre livres?) reçoit tour à tour le titre de Généalogies (Athénée, Stéphane de Byzance), d'Enquête(s) (historía[i]); Stéphane de Byzance, Strabon, Démétrius de Phalère) et d'Hérôologies (Harpocration). Le traité d'histoire locale de ce suggrapheùs pánu arkhaîos qu'est Antiochos de Syracuse selon Denys d'Halicarnasse (Ant. Rom. I, 12, 3) est désigné comme un traité sur la Sicile (Sikeliôtis suggraphé) par Pausanias (X, 11, 3). Quant à Diodore de Sicile (XII, 71, 2), il désigne ce même recueil écrit comme "enquête (historía) sur les affaires de Sicile ». Ce traité est sans doute distinct du Perì Italías dont on vient de mentionner la signature. Quant à Acousilaos d'Argos, son biographe lui attribue des Généalogies (Souda, s.v. Akousilaos, a 942 Adler) qui sont classées dans la catégorie générique de l'historía par Éphore (FrGrHist 70 F 20).

Quant à l'hypothèse d'une mythographie comme genre, la situation est fort bien illustrée par les témoignages relativement nombreux que nous avons sur le célèbre Phérécyde d'Athènes. Dénommé tour à tour historikós, historicus, historiográphos ou genealógos (selon la liste de témoignages donnée par R.F.: I, p. 272), sinon arkhaîos suggrapheús par Denys d'Halicarnasse (Ant. Rom. I, 13, 1, dans un passage que l'on va retrouver avec Antiochos de Sicile), Phérécyde se voit attribuer par la notice de la Souda (s.v. Pherekúdes, ph 214 Adler = test. 1 Fowler) la première rédaction d'un suggraphé en prose (pezôi lógoi). En général dénommé Historíai, sinon Théogonie, ce traité écrit en dix livres parcourt plusieurs grandes lignées hellènes, dans des récits généalogiques qui conduisent parfois depuis un fondateur divin ou héroïque jusqu'au présent : telle la généalogie de la famille des Philaïdes dont descend Miltiade. La citation que nous en avons (FGrHist $3 \mathrm{~F} 2$, dans un fragment qui est intégré à la collection de R.F. : voir le bon commentaire comparatif qu'il en donne [II, p. 474-478]) déroule la généalogie de la grande famille athénienne, au présent puis à l'aoriste narratifs, depuis l'ancêtre Philaios, le fils d'Ajax de Salamine, jusqu'à Miltiade, le fondateur du Chersonèse; le récit généalogique conduisait sans doute jusqu'à Cimon, l'homme politique contemporain de Phérécyde, à Athènes même ${ }^{5}$. C'est dire qu'il n'est pas possible de parler de «mythographie » ni comme genre ni même comme activité critique avant la fin $\mathrm{du} \mathrm{IV}^{\mathrm{e}}$ siècle. Pensons par exemple aux Tragoidoúmena d'Asclépiade de Tragilos (FGrHist 12 T 2). Élève d'Isocrate de même qu'Éphore et Théopompe, il travailla dans l'école d'Aristote, ce qui n'est pas un hasard ${ }^{6}$. En raison de ce travail critique, ce que nous identifions comme mythographie est toujours le lieu d'une interprétation, ne serait-ce que dans la confrontation des différentes versions livrées par une tradition extrêmement diversifiée.

\section{Un « style mythographique »?}

Donc, dans nos témoignages indigènes, on ne trouve ni mythographe comme fonction, ni mythographie comme genre, ni même comme forme discursive. 

style? Un registre singulier, comme l'indique R.F. dans une bonne étude consacrée à cette question ${ }^{7}$ ? Un style factuel, de l'ordre du résumé, sans intervention d'ordre énonciatif? "The newly-minded style of mythography» comme R.F. (II, p. 627-628) l'énonce à propos d'un fragment d'Acousilaos d'Argos (FgrHist. 2 F $22=$ fr. 22, 56-83 Fowler) qui raconte l'union de Kainé, la fille d'Élatos, avec Poséidon, puis la transformation de l'héroïne par le dieu en un homme invulnérable, pourvu d'une force surhumaine; il devint ainsi le roi des Lapithes et, conduisant la guerre contre les Centaures, il trouva la mort par la volonté de Zeus. Ce style mythographique de récits pratiquement réduits à leur intrigue et aux noms de leurs protagonistes serait significatif des « revolutionary implications of such fact-oriented works ». chez Hérodote, pourtant exclu (à tort) de la collection des fragments appartenant au genre supposé de la ( « early ») mythographie. Certes, les résumés mythographiques que donne l'enquêteur d'Halicarnasse au début de son lógos (I, 1-4) sont attribués aux lógioi des Perses : rapt d'Iô, la fille du roi d'Argos, par les Phéniciens; rapt par les Grecs d'Europe, la fille du roi de Tyr; puis rapt par ces mêmes Hellènes de Médée, la fille du roi d'Aia en Colchide ; enfin enlèvement d'Hélène par le fils de Priam Alexandre et donc guerre de Troie comme origine de l'inimité entre Perses et Hellènes. Cette série de rapts implique des franchissements territoriaux successifs de la limite entre Asie (représentée par les Perses) et l'Europe (défendue par les Grecs); cette séquence correspond à un enchaînement de ruptures de l'équilibre de la justice et par conséquent les causes des futures guerres médiques. Quant à Hérodote (I, 5, 3-7, 4), il recherche l'origine de cette haine dans le règne du roi de Lydie Crésus dont il fait remonter la généalogie non pas au retour des Héraclides, mais directement à un certain Alcée, fils d'Héraclès. Une analyse linguistique de détail montrerait que, dans ce double récit, les procédures sont très proches de celles du style « factuel » attribué par R.F. aux «mythographes» - on va y revenir.

\section{« Mythes » : Archaîa et palaiá}

17 Reste enfin la question des dénominations indigènes de ce que nous identifions comme «mythes », c'est-à-dire (pour faire bref...) des récits qui racontent les actions de protagonistes qui, avec le statut d'héroïnes et de héros, sont encore proches des dieux ; elles et ils évoluent dans des espaces qui coïncident en général avec ceux de la Grèce classique, mais dans un temps correspondant à l'ancienneté d'un pote qui, pour être chroniquement vague, n'en assume pas moins souvent une valeur fondatrice. On l'a répété à satiété : le terme grec mûthos ne renvoie pas, dans ses emplois préclassiques et classiques à ce que nous identifiions comme un mythe. Le mûthos correspond à un discours développé, argumenté et conçu pour être efficace, pour emporter la conviction. $\mathrm{Au}$ nombre des arguments soutenant le mûthos, avec la pratique correspondante du mutheîsthai, se trouve le récit héroïque avec sa valeur illustrative. La relation avec la situation présente est constitutive du mûthos. Ce n'est guère que chez Platon que le terme mûthos est employé, d'ailleurs en concurrence avec lógos (voir par exemple l'emploi de muthología et de muthologeîn), pour désigner un récit qui, mettant en scène divinités et figures hérö̈ques, comporte des invraisemblances et des incohérences d'ordre moral et non pas empirique ${ }^{8}$. 

qui nous donne la réponse: de même qu'Hérodote il désigne les événements de ce temps du tò pálai en tant que tà palaiá ou tà arkhaîa $a^{9}$ Ce n'est pas un hasard si un scholiaste a défini comme "archéologie» (littéralement: "le dire des temps du début ») le récit que fait Thucydide, au commencement de son traité, des événements qui anticipaient l'emprise politique, économique et militaire de l'Athènes contemporaine sur le bassin de la Mer Égée : de la première intervention d'Hellen pour rassembler les Grecs au combat naval le plus ancien qui opposa les Corinthiens aux Corcyréens, en passant par une première maîtrise colonisatrice des Cyclades par le roi de Crète Minos et naturellement par la guerre de Troie avec son protagoniste principal, Agamemnon. En raison de leur transmission par une tradition orale (comprise comme mnêma) et parce qu'elles nous sont connues par des poètes tel Homère ou par des logographes qui soucieux de séduire leur public les embellissent, ces actions des temps anciens doivent être soumises à l'examen critique (skopeîn) du rédacteur; leur historicité n'est néanmoins jamais mise en doute dans les réflexions présentées à ce propos (en I, 20,1-21).

19 Ne serait-ce qu'à ce titre, des extraits de l' «archéologie » de Thucydide, de même que toute la partie à la tournure "mythographique» qui ouvre l'Enquête d'Hérodote, méritaient de figurer dans la collection éditée et commentée par R.F. D'ailleurs, dans l'un des deux dialogues où Platon met en scène Hippias d'Élis, le sophiste dénomme arkhaiología (Hippias majeur, $285 \mathrm{~d}=$ FGrHist $6 \mathrm{~T} 3=86 \mathrm{~A} 11$ Diels-Kranz) les récits sur les héros, les hommes et les fondations de cités aux temps anciens (tò arkhaîon) avec lesquels il charme son public. Dans un fragment cité par Clément d'Alexandrie (Strom. VI, 15, 1 = FGrHist 6 F 4 = 86 B 6 Diels-Kranz), Hippias déclare son intention de composer (poiésomai) son propre récit (lógos) polymorphe aussi bien à partir de ce qui se trouve chez les poètes (tels Orphée, Musée, Hésiode et Homère) que dans les traités (suggraphaí) et des Grecs et des barbares. C'est précisément l'un des logographes que R.F. exclut de son corpus (I, p. XXXII-XXXIII)...

Par ailleurs, du côté des signifiants, Denys d'Halicarnasse (Ant. Rom. I, 28, 3) dénomme lógos le récit en partie généalogique par lequel Hellanicos de Lesbos (FGrHist $4 \mathrm{~F} 4=$ fr. 4 Fowler) rend compte de l'installation des Pélasges en Italie et de la fondation de Tursenia en Étrurie qui aurait donné son nom aux Tyrrhéniens. Et, selon le même Denys d'Halicarnasse (Ant. Rom. I, 13, 1 et 3), Antiochos de Syracuse lui-même (FGrHist $555 \mathrm{~F} 2$ = fr. 2 Fowler), dans la sphragís déjà alléguée, appelle arkhaîi lógoi les récits dont il va tirer son traité (sunégrapse) sur l'Italie. C'est l'installation des Oinôtroi, mentionnés plus haut, qui marque le début de ce "temps ancien» (tò palaión). C'est une peuplade dont Phérécyde d'Athènes, l'arkhaîs suggrapheús, présente aussi le héros éponyme Oinôtros dans l'un de ses nombreux parcours généalogiques (FGrHist 3 F $156=$ fr. 156 et test. 6 Fowler). On remarquera à ce propos que ce que R.F. identifie comme une première écriture "mythographique » est indissolublement lié à la forme du récit généalogique tel qu'il est attesté par exemple dans le Catalogue des femmes attribué à Hésiode. D'ailleurs, aussi bien le traité d'Hécatée (liste des témoignages chez R.F. I, p. 123) que celui d'Acousilaos d'Argos (test. 1 Fowler; cf. infra) portent en particulier le titre non pas de Muthographía, mais de Genealogíai ${ }^{10}$.

21 Pour ne prendre que ces deux exemples. 


\section{Du mûthos au lógos, malgré tout}

22 Les erreurs dans l'approche lexicale et sémantique de la mythographie sont celles qui, parallèlement au travail considérable et remarquable sur la «early Greek mythography ", marquent également la tentative de dater du milieu du ve siècle "the invention of mythology ». Se fondant sur une analyse des emplois en contexte des seuls termes mûthos et lógos, R.F. parvient à reconstruire une opposition dichotomique entre mythe et logos. Si les Grecs n'avaient pas la notion d'une «mentalité mythique », si donc ils ne reconnaissaient ni une pensée mythique, ni un passage du mythe à la raison, en revanche, avec l'aide des mythographes, ils auraient délimité un domaine de la mythologie qui s'opposerait au lógos comme le fictif au factuel: "The mythos/logos contrast, as one between imagination and reason, fictive and factual, is established in the fifth century, and linked to critical enquiry in both history and philosophy in ways that correspond clearly to common understandings of 'myth' and 'reason' in Western tradition ${ }^{11}$. Non seulement le terme mûthos est en concurrence au ve siècle avec d'autres termes (parmi lesquels celui de lógos!) pour désigner un récit (quelle qu'en soit la valeur de vérité); non seulement le signifié que nous donnons à notre mythe est saisi par des signifiants tels arkhaîa ou palaiá en Grèce classique; mais surtout mûthos par exemple chez les poètes tragiques (Euripide inclus) désigne tout discours argumenté (parfois à l'aide d'un récit).

C'est dire que la mythographie - et donc la «mythologie » - est, pour la période envisagée, un genre bien évanescent, sinon inexistant. C'est dire aussi que, du point de vue de leur pratique scripturaire, les rédacteurs concernés ne sont jamais dénommés mythographes; ils ne se considèrent ni ne sont considérés comme tels. Pour désigner cette pratique de consignation et de rédaction par écrit, la notion qui semble prévaloir est celle de l'enquête spatio-temporelle (historía) et, pour dénommer les praticiens euxmêmes, on recourt en général au terme de suggrapheús. Pourquoi dès lors, et à ce titre, l'exclusion de Xanthos de Lydie du corpus proposé par R.F.? L'historiographe figure pourtant dans la longue liste que donne Denys d'Halicarnasse (Thuc., 5, 1) d'arkhaîoi suggrapheîs ayant vécu avant la Guerre du Péloponnèse et jusqu'à l'âge de Thucydide aux côtés, pour les plus jeunes, d'Hellanicos de Lesbos ou de Xénomédès de Céos. Ce dernier est cité de manière extensive en distiques élégiaques par Callimaque (Aitia fr. 75, 54-55 Pfeiffer) ; racontant l'histoire d'Acontios et Cydippé, le poète alexandrin le présente comme «l'antique (arkhaîs) Xénomédès qui autrefois (pote) a consigné toute l'île (de Céos) dans une tradition de mythologue (muthológou mnêma) »!

Nous voilà confrontés aux deux autres délimitations arbitraires provoquées par la définition ad hoc d'une supposée mythographie grecque pertinente comme genre prosaïque pour la période choisie, soit la fin $\mathrm{du} \mathrm{VI}^{\mathrm{e}}$ siècle jusqu'au début du $\mathrm{IV}^{\mathrm{e}}-$ rappelons-le.

D'une part, du point de vue de l'espace géographique recouvert par les históriai grecques classiques, les peuples "barbares" sont exclus du corpus des «mythographes »; à l'exception des Amazones et des Hyperboréens sous prétexte que les unes et les autres sont de "pure creatures of Greek myth» (p. xxxII)! Double erreur dans la mesure où d'un côté les Grecs se définissent volontiers en continuité (les Égyptiens) ou par contraste (les Scythes) avec les différents modes (souvent fort civilisés) de la «barbarie ». Par ailleurs, en particulier chez Hérodote, le déroulement de l'histoire a une composante spatiale qui est constitutive; le sens qui est donné à 
l'action passée des hommes est attaché au franchissement des limites géographiques assurées par l'équilibre de la justice, lui-même garanti par Zeus. Dans l'Enquête, ce que l'on a longtemps considéré comme constituant des excursus ethnographiques sont, ne serait-ce que du point de vue narratif et énonciatif, des développements intégrés au lógos historique en cours de déploiement.

\section{Ni mythe, ni histoire !} seulement ce n'est que par inférence que l'on parvient à attribuer à Hérodote une distinction entre «temps des hommes » et "temps des dieux ». Si dans un passage constamment allégué (III, 112, 2), Minos est rejeté hors de la "génération dite humaine ", par contraste avec le tyran Polycrate de Samos, le roi de Crète n'est pas explicitement attribué à un «temps des dieux»; et cela d'autant moins qu'en particulier la confrontation avec la longue chronologie égyptienne conduit Hérodote à remonter jusqu'à la guerre de Troie, sinon à Héraclès, et à faire appel ainsi à des figures qui appartiennent au "temps des héros $»^{12}$. Mais surtout le début de l'histoire a été un sujet de décisions très différentes chez les successeurs des premiers historiographes. Selon Diodore de Sicile (IV, 1, 1-6), Éphore l'élève d'Isocrate (FGrHist 70 T 8), dans son entreprise d'écriture des actions universelles en plein $\mathrm{IV}^{\mathrm{e}}$ siècle, a délibérément sauté les « antiques mythologies » (tàs palaiàs muthologías) pour faire du retour des Héraclides le début de son enquête. De même ses contemporains Callisthène et Théopompe se seraient-ils abstenus de rendre compte de ces vieux récits (palaioì mûthoi). Quant à Diodore de Sicile lui-même, il consacre explicitement les trois premiers livres de son histoire aux mythologies des autres peuples en suivant leurs enquêtes sur les dieux; puis il décide de commencer sa propre histoire des Grecs avec Dionysos, en suivant les enquêtes des Hellènes sur des « temps anciens » (arkhaîoi khrónoi) qui comprennent les exploits des héros et des demi-dieux les plus en vue. Pour les poètes, puis les logographes de l'époque préclassique et $\mathrm{du} \mathrm{v}^{\mathrm{e}}$ siècle, l'âge des héros s'achève soit avec les dernières unions des dieux avec des mortelles, soit avec la guerre de Troie et les retours vers la Grèce ${ }^{13}$.

Secondement, de fixer arbitrairement la fin du temps des héros et par conséquent le domaine du «mythe » au retour des Héraclides rompt la continuité que les Grecs percevaient entre ce qui pour nous relève du temps des dieux et des héros, et ce qui appartiendrait au temps des hommes. N'oublions pas qu'au milieu du IV siècle, Isocrate considère que non seulement la guerre de Troie, mais aussi les Persiká représentent des palaiá ; dans le Panégyrique (66-70 et 54-60), l'éloge des vertus présentes d'Athènes et l'exposition de ses droits à l'hégémonie passent non seulement par l'évocation des batailles menées contre Darius puis Xerxès, mais aussi par l'engagement contre les Amazones, les filles d'Arès, et par la lutte du roi Érechthée contre le roi thrace Eumolpe, le fils de Poséidon : ces palaiá font partie des pátria. Muthologeîn, c'est, par le récit, aussi bien expliquer que légitimer. Ainsi dans le discours rédigé pour le roi de Sparte Archidamos (16-24), Isocrate remonte précisément au retour des Héraclides 
dans le Péloponnèse pour justifier les prétentions territoriales présentes du souverain lacédémonien du côté de la Messénie ${ }^{14}$.

\section{Du temps des origines au présent}

logographes choisissent un début, une origine, aussi bien du point de vue temporel que du point de vue spatial. Ainsi en va-t-il d'Acousilaos d'Argos, qu'intentionnellement j'ai à peine mentionné jusqu'ici ${ }^{15}$. Dénommé tour à tour par ceux qui le citent historikós, historiográphos et arkhaîos suggrapheús (cf. R.F. I, p. 1), le rédacteur place en tête de ses Généalogies une théogonie; selon le témoignage de Platon (Banquet, $178 \mathrm{ab}=$ Acousilaos test. 6 a Fowler), Chaos, puis Gé et Éros y détenaient un rôle primordial de même que dans la Théogonie d'Hésiode. Le premier homme mortel, c'est Phorôneus, qui deviendra le premier roi d'Argos (fr. 23a, b, c Fowler). Quant au héros éponyme Argos, le fils de la mortelle Niobé et de Zeus (fr. 25 Fowler), Acousilaos le disait «né de la terre » (fr. 27 Fowler). L'orientation focale du récit généalogique sur les figures fondatrices d'Argos induisent à penser qu'il se développait jusqu'à déboucher sur le passé proche ou le présent.

C'est le cas, on l'a vu, de la généalogie athénocentrée des Philaïdes telle qu'elle est déployée par Phérécyde d'Athènes jusqu'aux proches parents de Cimon, dans un but d'ordre assurément idéologique et politique. Dès lors, il est difficile de donner Phérécyde d'Athènes comme "the revolutionary who defined the genre of mythography" (R.F. II, p. 706). Il inaugurerait le "newly minded style of mythography» (II, p. 627) que l'on pourrait repérer également dans le fr. 22 d'Acousilaos d'Argos. Si Phérécyde " created the mythographical patrimony", si ses Enquêtes deviennent une "première encyclopédie » et une " œuvre de référence » (R.F. II, p. 707), c'est sans aucun doute à travers les usages postérieurs de récits généalogiques en prose qui assument souvent une dimension étiologique ${ }^{16}$. On l'a vu - l'argument du « just-the-facts style » n'est pas suffisant pour échapper à la conclusion que Rosalind Thomas tire du déploiement par Phérécyde de la généalogie de la famille athénienne des Philaïdes: "The family's impressive service to Athens is incorporated and hints of its tyrannical connections omitted, in what is clearly a political statement as well as family tradition ${ }^{17}$.

Si l'écriture du récit généalogique peut paraître en effet factuelle et « impersonnelle », elle est tout de même porteuse d'un redressement généalogique et d'une orientation énonciative en direction du présent. Selon Diodore de Sicile (XII, 71, 2 = test 3 Fowler), Antiochos de Syracuse conduisait son Enquête en neuf livres depuis le règne de Kôkalos, le roi premier des Sicanes, jusqu'au congrès de Géla où les cités de Sicile décidèrent de s'allier contre les Athéniens, soit en 424/5. R.F. lui-même reconnaît (II, p. 190 et 633) que l'origine particulière qu'Antiochos attribue à Métaponte est sans doute dirigée contre Athènes. D'ailleurs bien habile serait celle ou celui capable d'identifier dans la chronique du Marbre de Paros des traits stylistiques permettant de distinguer une période mythique d'une période historique. La conduite narrative et énonciative de la chronique déroule sur le même mode les événements qui ont marqué l'histoire politique et culturelle d'Athènes depuis le règne du roi primordial Cécrops jusqu'au moment de la consécration de l'inscription à Paros en $264 / 3$ en passant par exemple par le règne de Minos, l'intervention de Déméter en Attique, la bataille de Marathon, la mort de Sophocle ou le début du règne d'Alexandre ${ }^{18}$. 
C'est dans cette perspective de continuité entre temps des héros et temps des hommes pragmatiquement orientée sur le présent que s'inscrit l'Attikè suggraphé d'Hellanicos de Lesbos. Les quelques rares fragments qui nous sont parvenus de cette première Atthís évoquent non seulement la fille de Cécrops Agraulos (fr. *38 Fowler), le roi légendaire Mounychos (fr. 42 a et $b$ Fowler) ou le fils de Poséidon Hippothoon, le héros éponyme de la tribu attique des Hippothoontides (fr. 3, 43 Fowler) ; mais Thucydide lui-même (I, $97,1)$ confronte la version détaillée qu'il donne de la montée en puissance d'Athènes entre les guerres médiques et le début de la guerre du Péloponnèse au résumé insuffisant et chronologiquement inexact qu'en fait Hellanicos dans une Attikè suggraphé (c'est le titre donné par le contemporain Thucydide) qui devait conduire jusqu'à la fin de la guerre du Péloponnèse en $404 / 3{ }^{19}$. La distinction naturalisante moderne entre mythe et histoire, fermement et obstinément réaffirmée par R.F. (II, p.

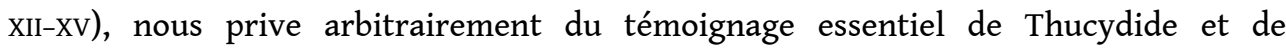
l'important fragment FGrHist $4 \mathrm{~F} 49$ ! Elle exclut toute réflexion sur la fonction et la pragmatique de traités écrits qui s'inscrivent, de même que les lógoi d'Hérodote pour l'histoire et la géographie humaine, dans les vastes interrogations et enquêtes critiques et pratiques; en sont témoins pour nous les médecins hippocratiques pour la conception physiologique de l'homme, les sophistes pour la réflexion sur la langue et la rhétorique, les sages préplatoniciens pour la cosmologie et l'anthropologie. En Grèce classique, l'enquête sur le passé héroïque des cités et de l'Hellade ne se conçoit et ne se comprend qu'à partir de la conjoncture historique et culturelle du présent. Elle n'est jamais pure érudition.

\section{L'éditon et ses deux commentaires}

Les conséquences de tous ces malentendus lexicaux et sémantiques? Divisé en deux volumes publiés à treize ans de distance, l'ouvrage de R.F. est composé de quatre parties : une introduction où se trouve légitimé le choix des historiographes et des textes édités et commentés (avec les justifications complémentaires apportées dans l'introduction au volume II, p. XII-XVII) ; l'édition philologique des témoignages et des fragments des rédacteurs sélectionnés (avec un appendice où sont consignées les leçons des manuscrits des scholies homériques qui n'affectent pas le contexte des fragments cités) ; un commentaire développé, lui-même divisé en deux parties : un «mythological commentary" et, plus succinct, un "philological commentary». Le tout est assorti d'une longue bibliographie et d'indices aussi précieux qu'ils sont rendus indispensables pour la consultation d'un ouvrage de plus de mille pages, à l'impression serrée.

Avec les limites indiquées quant à la période couverte, quant au contenu admis comme mythique et quant à la définition d'un genre mythographique inexistant, le texte des témoignages et des fragments édités reprend en gros celui des FGrHist édités par Felix Jacoby. L'ordre d'édition des fragments suit l'ordre alphabétique des rédacteurs choisis, à l'écart de tout classement chronologique; et pour chaque historiographe, la numérotation des témoignages et des fragments suit celle adoptée par Jacoby, avec les exceptions signalées dans une table de confrontation entre les deux éditions (I, p. 382388). S'y ajoutent les quelques très rares fragments papyrologiques publiés entretemps (voir la liste donnée dans l'index fontium, I, p. 397-398).

Le texte a été revu avec un remarquable souci d'exhaustivité et d'acribie philologiques, offrant un apparat critique aussi bien pour chaque fragment cité que pour le texte 
citant. Le commentaire quant à lui rompt avec la tradition de l'exégèse linéaire anglosaxonne qui associe sous les mêmes lemmes et dans les mêmes rubriques remarques de philologie, investigations d'ordre sémantique (en particulier par la confrontation avec des usages parallèles) et tentatives d'interprétation. Restreint aux questions d'établissement du texte, le "Philological commentary » offre de plus pour chaque auteur une présentation fort utile d'ordre bio-bibliographique et une liste des traits linguistiques particuliers aux fragments conservés.

Quant aux remarques développées de contenu, elles sont consignées dans le très long et surprenant "Mythological commentary» qui précède le commentaire philologique. Il constitue de fait une monographie de plus de six-cents pages sur les arkhaîa et les palaiá qui ont été réorganisés, réinventés, narrés et consignés par écrit par les premiers historiographes dans leurs traités généalogiques et historiques. Ce " commentaire » est lui-même articulé en grandes rubriques qui suivent le développement aussi bien des grandes familles héroïques panhelléniques, des traditions héroïques locales et des grandes sagas dans lesquelles certains de leurs protagonistes sont engagés. De la théogonie (Béance et ses descendants, Okéanos et ses enfants, Prométhée, Typhon, les Courètes, les Cyclopes, certains des dieux olympiens, etc.) jusqu'aux migrations (ioniennes, doriennes, éoliennes), en passant par les premiers peuplements pré-grecs (par les Pélasges, les Lélèges, voire les Dryopes) et naturellement par Héraclès et ses douze travaux et par ses fils les Héraclides. Deucalion et Pyrha avec leurs descendants (Hellen, per exemple) sont évidemment de la partie ainsi que les Éolides (Mélampous et les Prœtides), les Argonautes (la toison d'or) et les Inachides (avec la généalogie d'Iô). Le cycle thébain aussi bien que le cycle troyen y ont leur part au même titre que l'histoire locale de l'Attique, par exemple. Étant donné le caractère extrêmement fragmentaire de notre tradition, la confrontation avec une historiographie beaucoup plus tardive telle celle de Diodore de Sicile ou avec un ouvrage de mythographie à proprement parler telle la Bibliothèque attribuée à Apollodore s'avère indispensable.

En raison de la confrontation précise, soignée et exhaustive avec différentes traditions, ce « commentaire » peut offrir un usage instrumental complémentaire à celui du Early Greek Myth de Timothy Gantz ${ }^{20}$. Il n'en reste pas moins que si le recueil des fragments des rédacteurs et logographes est fondée sur le postulat pour le moins contestable de l'existence à l'époque classique d'une mythographie comme genre, le commentaire repose sur celui de l'existence d'un domaine du mythe dont la sémantique et les limites sont des plus floues. Ni l'une, ni l'autre ne correspondent à des catégories contemporaines des rédacteurs édités et commentés.

Les deux volumes de Early Greek Mythography nous offrent donc un instrument de travail sans doute très précieux, mais à utiliser avec beaucoup de circonspection. En dépit de son incontestable et admirable valeur du point de vue d'une érudition exhaustive et fiable, une telle collection ne pourra que continuer à entretenir, parmi les lectrices et lecteurs contemporains, les habituels malentendus sur mythologie et mythographie grecques. Ne serait-ce que par son intitulé, cette édition commentée ne tient aucun compte de la relation de l'hypothétique mythographie grecque avec les formes de l'histoire poétique. Cette relation semble pourtant encore bien attestée au I ${ }^{\text {er }}$ s. de notre ère dans une inscription d'Amorgos (IG XII 7, 273): le texte dédicatoire présente un certain Aristogénès comme le « mythographe d'Apollon et des Muses »! 


\section{NOTES}

1. .Pour un probable emploi du terme muthográphos dans le P.0xy. 4458 pour désigner Poséidonios, cf. R.F. I, p. XXVII, n. 1.

2. .Les problèmes posés par le recueil de Palaiphatos sont rappelés en particulier par A. SANTONI, Palefato. Storie incredibili, Pisa, ETS, 2000, p. 37-42; sur la préface du traité, voir G. HAWES, Rationalizing Myth in Antiquity, Oxford, OUP, 2014, p. 37-48. À ce propos, cf. R.F. II, p. XIV, n. 3.

3. .Pour quelques éléments d'une histoire du concept moderne de "mythographie ", voir J.N. BREMMER, «Local Mythography: The Pride of Halicarnassus », in S.M. TRZASKOMA, R.S. SMITH (éd.), Writing Myth. Mythography in the Ancient World, Leuven, Peeters, 2013, p.55-74 (55-56, avec bibliographie).

4. .Je me permets de renvoyer ici à l'étude proposée de cette "signature » d'Hécatée dans Mythe et histoire dans l'Antiquité grecque. La création symbolique d'une colonie, Paris, Les Belles Lettres, $2011^{2}$, p. 49-51.

5. .Pour le détail, voir la bonne étude de R. Thomas, Oral Tradition and Written Record in Classical Athens, Cambridge, CUF, 1989, p. 161-173. Pour les relations avec la poésie épique, voir l'étude récente de R.P. MARTIN, « The 'myth before the myth began' ", in J. FALAKY (éd.), Writing down the Myths, Turnhout, Brepols, 2013, p. 45-66.

6. .On verra à ce propos les premières pages de l'excellente synthèse offerte par E. PELLIZER, « La mitografia », in G. CAMBIANo (éd.), Lo spazio letterario della Grecia antica, vol. I. 2, Roma, Salerno Editrice, 1993, p. 283-303 ; c'est une étude qui manque dans la longue bibliographie offerte par R.F. II, p. 755-799.

7. . « How to Tell a Myth: Genealogy, Mythology, Mythography », Kernos 19 (2006), p. 35-46. Dans ce même volume collectif consacré aux formes du catalogue et de la généalogie dans la narration des mythes grecs, la très brève étude comparative que j'ai proposée (p. 23-29) entre la version poétique que nous donne Hésiode dans la Théogonie (132-138) et la version mythographique de la Bibliothèque attribuée à Apollodore ( $\mathrm{I}, 1,3)$ pour la descendance de Gé et d'Ouranos montre qu'audelà de quelques différences formelles relatives à la diction épique, la procédure de développement narratif est fondamentalement identique.

8. Références et nombreuses indications bibliographiques à ce propos dans l'étude citée à la note 1, p. 44-46 et 256-262.

9. .Encore une fois je fais référence à l'étude sémantique que j'ai proposée de ces deux termes dans «La fabrication historiographique d'un passé hérö̈que en Grèce classique : archaîa et palaiá chez Hérodote », Ktema 31 (2006), p. 39-49.

10. Sur le développement généalogique à partir de la forme du catalogue chez des «mythographes» avant la lettre, on pourra se référer à l'étude récente de Ch. DELATTRE, «Pentaméron mythographique. Les Grecs ont-ils écrit leurs mythes ? », Lalies 33 (2013), p. 77-170 (en particulier p. 105-110 et 117-123) ; voir aussi l'importante contribution de Ch. JACOB, « L'ordre généalogique entre le mythe et l'histoire ", in M. DETIENNE, Transcrire les mythologies, Paris, Albin Michel, 1994, p. 169-202 (qui ne figure pas non plus dans la bibliographie donnée par R.F.).

11. .R.L. FOWLER, « Mythos and logos ", JHS 131 (2011), p. 45-66, une étude marquée par les erreurs de méthode linguistique relevées dans Qu'est-ce que la mythologie grecque ?, Paris, Gallimard, 2015, p. 15-17.

12. .Voir à nouveau les références bibliographiques que j'ai données à propos de cette distinction discutable dans l'op. cit. note 1, p. 65-66 (avec note 71).

13. .On verra en particulier l'étude de G. TOSETTI, « La dernière génération héroïque : un parcours historico-religieux et sémio-narratif, d'Hésiode au ps.-Apollodore », Kernos 19 (2006), p. 113-130. 
Pour Diodore de Sicile, voir S. SAIID, «Entre mythographie et histoire : le traitement du mythe dans la Bibliothèque historique de Diodore de Sicile», in E.K. EMILSSON, A. MARAVELA, M. SKOIE (éd.), Paradeigmata. Studies in Honour of Ølvind Andersen, Athens, Norwegian Insitute, 2014, p. 67-86.

14. Pour le détail et les références bibliographiques de rigueur, je renvoie à mon étude « Mûthos, lógos et histoire. Usages du passé héroïque dans la rhétorique grecque », L'Homme 147 (1998), p. 127-149.

15. .Là encore référence à une étude personnelle : «Les fonctions généalogiques : Acousilaos d'Argos et les débuts de l'historiographie grecque », Europe 945-946 (2008), p. 87-108.

16. .En se référant en particulier aux travaux de Jordi PÀMIAS (cf. Ferecides d'Atenes. Històries, 2 vol., Barcelona, Fundació Bernta Metge, 2 vol.), DelATTRE, o.c. (n. 9), p. 111-117, relève à juste titre que le texte des fragments cités a été sans doute l'objet de reprises successives et ne renvoie donc qu'indirectement à l'original. On se référera à ce propos, pour l'histoire de Thèbes, à la bonne étude de D.W. BERMAN, «Greek Thebes in the Early Mythographic Tradition », in Trzaskoma Smith, o.c. (n. 3), p. 37-53

17. Dans le travail cité à la note 4, p. 173 ; voir à ce propos le commentaire de R.F. lui-même, II, p. 475-478 (avec note 85 !). Sur la continuité des récits généalogiques à apparence mythographique jusque dans le présent, voir encore JACOB, o.c. (n. 9), p. 193-199; dans l'étude compréhensive récente qu'il consacre aux différents modes de la mythographie, DELATTRE, o.c. (n. 10), p.117-126, fait la démonstration en creux que ni Acousilaos d'Argos, ni Phérécyde d'Athènes ne sont des mythographes.

18. J'entends y revenir dans une autre étude. On remarquera à ce propos que le texte mythographique et épigraphique dénommé Éloge d'Halicarnasse devait s'inscrire dans une même logique : voir à ce propos l'étude de Bremmer citée à la note 3 (avec la bibliographie suscitée par la nouvelle inscription de Salmakis), qui en conclut que " (ancient mythography) was not just an academic subject. It always served certain interests, be it local prestige, literary exprertise or the acquisition of cultural capital » (72).

19. Sur l'Atthis d'Hellanicos de Lesbos et sur cette hypothèse chronologique, voir les références données par S. HORNBLOWER, A Commentary on Thucydides. Volume I. Books I-III, Oxford, Clarendon Press, 1991, p. 147-148, ainsi que la mise en contexte proposée par F. HARTOG, Évidence de l'histoire. Ce que voient les historiens, Paris, Éditions de l'EHESS, 2005, p. 66-74.

20. .T. GANTZ, Early Greek Myth. A Guide to Literary and Artistic Greek Sources, Baltimore/London, The Johns Hopkins University Press, 1993 (trad. française sous le titre Mythes de la Grèce archaïque, Paris, Belin, 2004).

\section{AUTEURS}

\section{CLAUDE CALAME}

École des Hautes Études en Sciences Sociales, Paris 